\title{
Ultrabroadband THz spectroscopic investigation of As2S3
}

Zalkovskij, Maksim; Malureanu, Radu; Novitsky, Andrey; Savastru, Dan ; Popescu, Aurelian ; Lavrinenko, Andrei; Jepsen, Peter Uhd

Published in:

CLEO Technical Digest

Publication date:

2012

Document Version

Publisher's PDF, also known as Version of record

Link back to DTU Orbit

Citation (APA):

Zalkovskij, M., Malureanu, R., Novitsky, A., Savastru, D., Popescu, A., Lavrinenko, A., \& Jepsen, P. U. (2012). Ultrabroadband THz spectroscopic investigation of $\mathrm{As}_{2} \mathrm{~S}_{3}$. In CLEO Technical Digest (pp. CM1L.2). Optical Society of America.

\section{General rights}

Copyright and moral rights for the publications made accessible in the public portal are retained by the authors and/or other copyright owners and it is a condition of accessing publications that users recognise and abide by the legal requirements associated with these rights.

- Users may download and print one copy of any publication from the public portal for the purpose of private study or research.

- You may not further distribute the material or use it for any profit-making activity or commercial gain

- You may freely distribute the URL identifying the publication in the public portal

If you believe that this document breaches copyright please contact us providing details, and we will remove access to the work immediately and investigate your claim. 


\title{
Ultrabroadband THz spectroscopic investigation of $\mathrm{As}_{2} \mathrm{~S}_{3}$
}

\author{
Maksim Zalkovskija ${ }^{\mathrm{a}}$ Radu Malureanu ${ }^{\mathrm{a}}$, Andrey Novitsky ${ }^{\mathrm{a}}$, Dan Savastru $^{\mathrm{b}}$, Aurelian Popescu ${ }^{\mathrm{b}}$, \\ Andrei V. Lavrinenko a and Peter Uhd Jepsen ${ }^{\mathrm{a}}$ \\ a) DTU Fotonik, Technical University of Denmark, DK-2800 Kongens Lyngby, Denmark \\ b) National Institute of R\&D for Optoelectronics INOE 2000, Romania \\ mzal@fotonik.dtu.dk
}

\begin{abstract}
We perform ultrabroadband $\mathrm{THz}$ spectroscopy of the dielectric function of arsenic trisulfide $\left(\mathrm{As}_{2} \mathrm{~S}_{3}\right)$. We observe the transition from universal scaling of the absorption at low frequencies to medium- and short-range-order at higher frequencies.

OCIS codes: (300.6270) Spectroscopy, far infrared; (300.6495) Spectroscopy, terahertz; (160.2750) Glass and other amorphous materials.
\end{abstract}

\section{Introduction}

Non-crystalline materials are a class of materials which encompass liquids, semiconductors and amorphous solids. While long-range order is absent in these materials, they are still characterized by a certain amount of local order. For instance, liquid water consists of identical water molecules with an average ordering of a local environment in the dynamic and random global environment. This mixture of order and disorder is reflected in the broadband absorption spectrum of liquid water which is dominated by the global relaxational dynamics at low frequencies (up to few THz), vibrational modes of the intermolecular structures at intermediate frequencies $(5-15 \mathrm{THz})$, and molecular vibrations at high frequencies (up to approximately $100 \mathrm{THz}$ ) [1]. A similar example is amorphous molecular glasses, where individual molecules (for instance, in the chalcogenide soft glass arsenic trisulfide) are arranged with a certain amount of local and medium-range order, while the global network of the molecules is arranged in a random fashion. In contrast to liquids, the dielectric properties in amorphous glasses are dominated by a continuous distribution of vibrational modes with universal properties in the low $\mathrm{THz}$ range [2], while the relaxation dynamics is expected at much lower frequencies due to the rigid network.

Here we discuss the limits of the validity of the universal absorption features in disordered materials [2] determining as an example the ultrabroadband dielectric function of the soft glass $\mathrm{As}_{2} \mathrm{~S}_{3}$, and identify regions of high transparency in the $\mathrm{THz}$ range.

\section{THz spectroscopy measurements}

Most terahertz time-domain spectroscopy (THz-TDS) studies to date have been focused on the region below $3 \mathrm{THz}$ due to instrumentation limitations (electro-optic generation and detection in ZnTe crystals or photoconductive switches). This narrow bandwidth of spectroscopic systems naturally enforces certain limitations to the interpretation of measured spectra, in particular in the case of broad, rather featureless absorption profiles. Extended bandwidth measurements have proven to be much more valuable, as exemplified by references [3-5].

Here we use a THz-TDS system which employs the air plasma generation of ultrashort THz transients [6,7] in combination with the air biased coherent detection (ABCD) of the $\mathrm{THz}$ transients [8].

Both the generation and detection processes are based on the four-wave mixing in air. Similar to any other frequency conversion process, the bandwidth of the process is always limited by the bandwidth of the laser source and the phase matching conditions. With air as the nonlinear medium the phase matching conditions are perfect over an extremely broad bandwidth range, and therefore the bandwidth of the generation and detection processes is in practice limited only by the laser bandwidth.

We use a transform-limited 35-fs laser pulse from a standard regenerative amplifier system (SpectraPhysics Spitfire), resulting in $\mathrm{THz}$ transients with a spectral coverage from 1 to $40 \mathrm{THz}$ and $\mathrm{THz}$ pulse duration of less than 50 fs. In combination with a standard THz-TDS system based on photoconductive switches we can perform quantitative THz-TDS on absorptive materials in the $0.05-20 \mathrm{THz}$ range, thus simultaneously covering both the disorder region below the Ioffe-Regel transition and the order region at higher frequencies. 

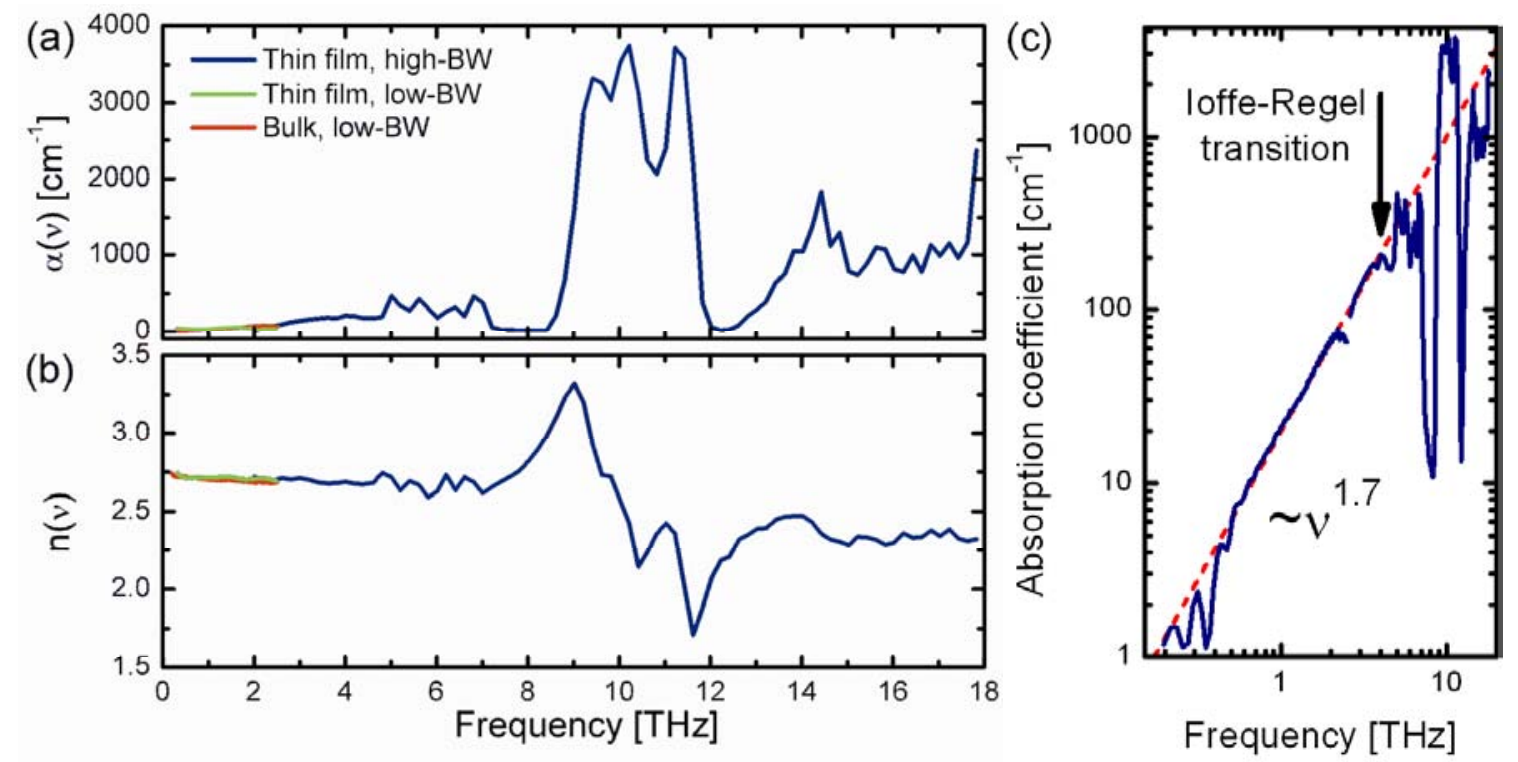

Fig. 1. (a) Absorption coefficient of $\mathrm{As}_{2} \mathrm{~S}_{3}$ at room temperature. Data represented by the green and red curves are recorded with a lowbandwidth THz-TDS system, data represented by the navy curve are recorded with a high-bandwidth THz-TDS system. (b) Index of refraction of $\mathrm{As}_{2} \mathrm{~S}_{3}$. (c) Log-log plot of the absorption coefficient, demonstrating the transition from disorder (power-law scaling) to order (isolated vibrational modes).

\section{Results}

The absorption coefficient and index of refraction of the arsenic trisulfide is shown in Fig. 1.

The data is the result of recordings on several samples with different thicknesses using two different THz-TDS spectroscopy setups. High-bandwidth measurements were performed on three different thin-film sample thicknesses from 3 to $14 \mu \mathrm{m}$; low-bandwidth measurements were performed on a $14 \mu \mathrm{m}$-thin film and a bulk $(0.85 \mathrm{~mm})$ sample.

We observe several interesting features in the resulting refractive index spectra of $\mathrm{As}_{2} \mathrm{~S}_{3}$. At low frequencies we observe the expected broad, monotonously increasing absorption, which is a general signature of disordered materials [2]. As shown in Fig. 1(c) the characteristic power-law behavior extends up to $4 \mathrm{THz}$ can be used for describing the material's behavior. In the frequency range 4-7 THz we observe highly reproducible absorption features originating from medium-range order in the glass, followed by three strong absorption lines in the 10-12 $\mathrm{THz}$ range and a peak at $14.5 \mathrm{THz}$, originating from intramolecular vibrations in the $\mathrm{As}_{2} \mathrm{~S}_{3}$ molecular unit.

$\mathrm{As}_{2} \mathrm{~S}_{3}$ has very high third-order nonlinearity in the mid-infrared region, and there is a large interest in extending nonlinear optics based on soft glasses into the far infrared. For the future development of nonlinear $\mathrm{THz}$ optics we find it very promising that there are two clear transparency regions in the frequency range near $8 \mathrm{THz}$ and $12.3 \mathrm{THz}$.

\section{References}

[1] U. Møller, D. G. Cooke, K. Tanaka and P. U. Jepsen, "Terahertz reflection spectroscopy of Debye relaxation in polar liquids [Invited]," J. opt. Soc. Am. B 26, A113-A125 (2009).

[2] S. N. Taraskin, S. I. Simdyankin, S. R. Elliott, J. R. Neilson and T. Lo, "Universal features of terahertz absorption in disordered materials," Phys. Rev. Lett. 97, 055504 (2006).

[3] A. Pashkin, C. Kübler, H. Ehrke, R. Lopez, A. Halabica, R. F. Haglund, Jr., R. Huber and A. Leitenstorfer, "Ultrafast insulator-metal phase transition in VO2 studied by multiterahertz spectroscopy," Phys. Rev. B 83, 195120 (2011).

[4] A. Pashkin, M. Porer, M. Beyer, K. W. Kim, A. Dubroka, C. Bernhard, X. Yao, Y. Dagan, R. Hackl, A. Erb, J. Demsar, R. Huber and A. Leitenstorfer, "Femtosecond Response of Quasiparticles and Phonons in Superconducting YBa2Cu3O7-delta Studied by Wideband Terahertz Spectroscopy," Phys. Rev. Lett. 105, 067001 (2010).

[5] R. Huber, F. Tauser, A. Brodschelm, M. Bichler, G. Abstreiter and A. Leitenstorfer, "How many-particle interactions develop after ultrafast excitation of an electron-hole plasma," Nature 414, 286-289 (2001).

[6] D. J. Cook and R. M. Hochstrasser, "Intense terahertz pulses by four-wave rectification in air," Opt. Lett. 25, 1210-1212 (2000).

[7] X. Xie, J. M. Dai and X. C. Zhang, "Coherent control of THz wave generation in ambient air," Phys. Rev. Lett. 96, 075005 (2006).

[8] J. Dai, X. Xie and X. C. Zhang, "Detection of broadband terahertz waves with a laser-induced plasma in gases," Phys. Rev. Lett. 97, 103903 (2006).

[9] P. U. Jepsen, D. G. Cooke and M. Koch, "Terahertz spectroscopy and imaging - Modern techniques and applications," Laser Photon. Rev. 5, 124-166 (2011). 